\title{
Total Electron Yield Mapping of Electronic Device Features via Measurement of X- Ray Beam-Induced Currents
}

William A. Hubbard ${ }^{1}$, Jared J. Lodico ${ }^{1}$, Brian Zutter ${ }^{1}$, David Shapiro ${ }^{2}$, Yuan Hung Lo ${ }^{1,3}$, Arjun Rana ${ }^{1}$, Drew Morrill ${ }^{4}$, Christian Gentry ${ }^{4}$, Ho Leung Chan ${ }^{1}$, and B. C. Regan ${ }^{1}$

1. Department of Physics \& Astronomy and California NanoSystems Institute, University of California, Los Angeles, CA, USA.

2. Advanced Light Source, Lawrence Berkeley National Laboratory, Berkeley, CA, USA.

${ }^{3 .}$ Department of Bioengineering, University of California, Los Angeles, CA, USA.

4. Department of Physics and JILA, University of Colorado and NIST, Boulder, CO, USA.

In scanning transmission X-ray microscopy (STXM), a focused X-ray beam is scanned across a sample, and detector signals are associated with the beam position to form images. In standard STXM the detectors count X-rays. In non-standard STXM techniques such as X-ray beam-induced current (XBIC) and total electron yield (TEY) imaging, the "detector" counts electrons, i.e. electrical currents generated in or by the sample. XBICs, such as those generated by electron-hole pair separation, provide information about charge carriers and local electric fields, and are typically collected from electrical leads on the sample [1]. TEY measures the total emission of (e.g. secondary) electrons by collecting the beam-induced currents in the entire sample [2].

Here we describe a STXM technique which measures the total current arising from electron emission, as in TEY imaging, but does so in a localized region defined by an electrical lead, as in XBIC. Based on an electron beam-induced current (EBIC) imaging technique recently developed for scanning transmission electron microscopy (STEM) [3], this TEY-XBIC STXM technique has several advantages: 1) it provides differential contrast unavailable to conventional TEY measurements, 2) it enables correlative STXM/STEM measurements of electronic features in devices, and 3) it circumvents some of the technical challenges of performing TEY measurements in conjunction other X-ray imaging modes.

The X-ray experiments were performed at the COSMIC beamline at the Advanced Light Source at Lawrence Berkeley National Lab, which has soft X-ray STXM capabilities. At this beamline samples are inserted into the vacuum chamber on standard TEM sample holders, using a TEM CompuStage (FEI/Philips) as the load-lock system. Commercially available in situ TEM holders thus allow for STXM of devices subject to electrical bias, heating, and liquid and gas environments. For this experiment, microfabricated X-ray-transparent devices were held and electrically contacted using a TEM biasing sample holder (Hummingbird Scientific).

A $100 \mathrm{~nm} \times 1 \mu \mathrm{m} \mathrm{Al}$ wire patterned on a $20 \mathrm{~nm} \mathrm{Si} \mathrm{N}_{4}$ membrane was biased until it failed via electromigration, causing a gap in the wire (Fig. 1, HAADF). In Fig. 1 (upper right), the bright SEEBIC signal maps a region of $\mathrm{Al}$ which is connected to the intact right side of the wire, including a thin protrusion to the left of the wire gap. Surprisingly, the electrical connectivity revealed by SEEBIC does not mirror the mechanical connectivity implied by HAADF: the thin protrusion appears to be electrically isolated ( $>\mathrm{G} \Omega$ ) from the adjacent aluminum.

Images of the same device analogous to these STEM images were collected with soft X-ray STXM (Fig. 1, bottom row). The photon energy was tuned to $1565 \mathrm{eV}$ to maximize absorption at the Al K-edge. The STXM resolution is limited by the X-ray spot size to $40 \mathrm{~nm}$; however, a ptychographic reconstruction of 
coherent diffraction patterns (Fig. 1, lower left) improves the resolution to $\sim 10 \mathrm{~nm}$. Both X-ray images were acquired simultaneously. Here, the TEY-XBIC shows the same bright (i.e. conducting and connected) protrusion across the ptychographically resolved gap in the wire. The X-ray absorption (and the XBIC) decreases drastically away from resonance (not shown), indicating the protrusion is $\mathrm{Al}$.

For devices, the differential contrast provided by isolating electron yield measurements to a single (or, individually, multiple) electrodes provides maps related to electronic structure. As examples, X-ray imaging of switching of oxidation states in resistive memory devices via spectroscopy [4] or magnetic features in magnetic memory devices via dichroism [5] can be directly correlated to corresponding electronic changes (e.g. conductivity). TEM experiments on the same devices can be performed without removing the sample from the holder for direct comparison of nanoscale features to spectroscopic and dichroic features. This technique also enables TEY imaging without modifications to the beamline that are often incompatible with high-resolution STXM [2], e.g. biasing the zone plate [6].

\section{References:}

[1] OF Vyvenko et al., Journal of Applied Physics, 91 (2002), p. 3614-3617.

[2] D Nolle et al., Microscopy and Microanalysis, 17 (2011), p. 834-842.

[3] WA Hubbard et al., Physical Review Applied, 10 (2018), 044066.

[4] A Koehl et al., APL Materials, 1 (2013), p. 042102.

[5] DP Bernstein et al., Physical Review B, 83 (2011), p. 180410.

[6] This work has been supported by National Science Foundation STC award DMR-1548924

(STROBE) and by the UCLA PSEIF. The authors acknowledge the use of instruments at the EICN supported by NIH 1S10RR23057 and the CNSI at UCLA, and the COSMIC beamline at Lawrence Berkeley National Laboratory, supported by the U.S. Department of Energy under contract number DEAC02-05CH11231.

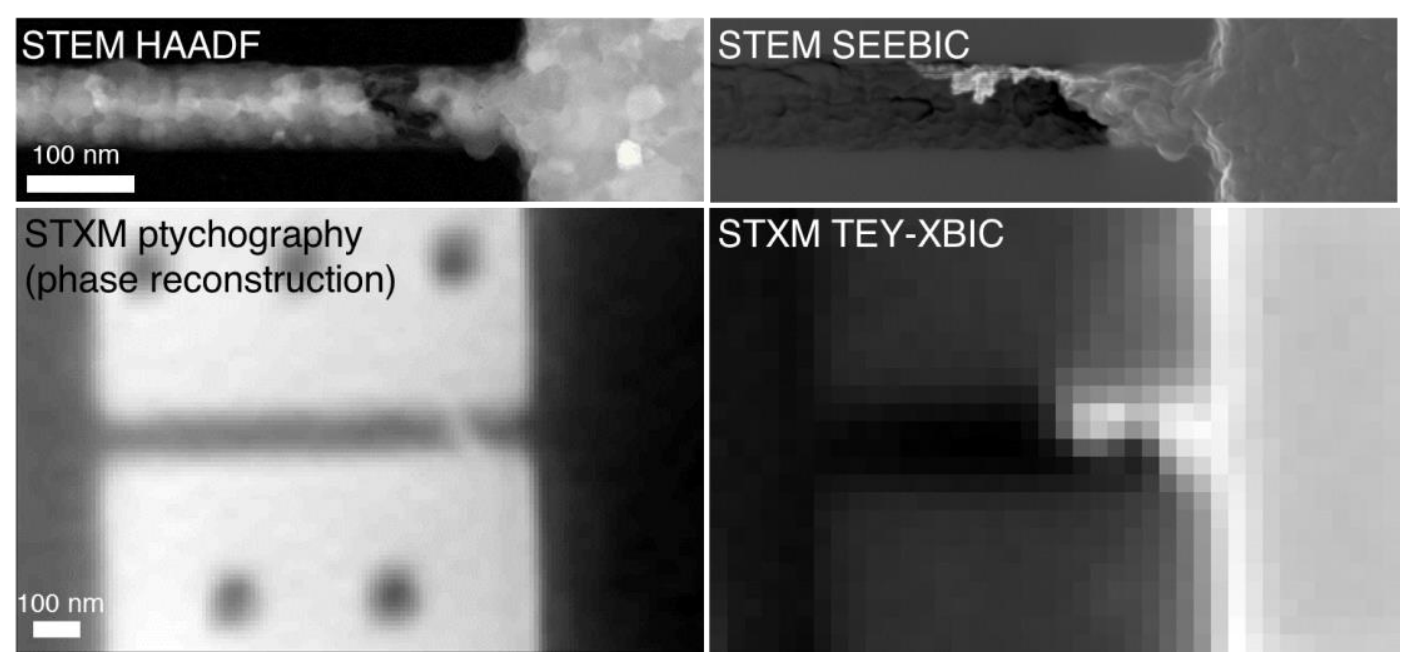

Figure 1. STEM HAADF and SEEBIC images and STXM ptychography and TEY-XBIC images of a damaged Al wire. The (upper) STEM images were acquired with $80 \mathrm{kV}$ electrons and the (lower) X-ray images were acquired with $1565 \mathrm{eV}$ photons. In each beam-induced current image, current is measured from the right $\mathrm{Al}$ electrode and the left electrode is held at ground. Bright/dark contrast in the induced current images corresponds to positive/negative (hole/electron) current. 